\title{
Tratamento com a Técnica de Duplo Cateter-Guia de Perfuração Coronária Tipo III
}

\author{
Evandro Martins-Filho1, Tarcísio Campostrini Borghi Jr. ${ }^{2}$, Marcelo Aguilar Puzzi³ \\ Ricardo Costa ${ }^{4}$, Dimytri Siqueira ${ }^{5}$, Rodolfo Staico ${ }^{6}$, Alexandre Abizaid ${ }^{7}$
}

\section{RESUMO}

A perfuração coronária é uma complicação rara da intervenção coronária percutânea e potencialmente catastrófica. Pode levar ao derrame pericárdico, com ou sem tamponamento cardíaco, e, se não diagnosticada com precocidade e tratada adequadamente, pode levar ao óbito. Relatamos o caso de uma intervenção coronária percutânea complicada com perfuração coronária, rapidamente tratada com reversão da anticoagulação, insuflação prolongada com balão e implante de stent recoberto pela técnica do duplo cateter-guia.

DESCRITORES: Intervenção coronária percutânea. Stents. Cateteres cardíacos.

A perfuração coronária (PC) é uma complicação infrequente nas intervenções coronárias percutâneas (ICP), podendo levar a derrame pericárdico e tamponamento cardíaco. Quando não diagnosticada e tratada a tempo, pode levar ao óbito. A incidência das PCs associadas à ICP é de, aproximadamente, 0,1 a $0,6 \%$, e sua ocorrência está ligada a lesões complexas, principalmente aquelas com calcificação intensa e oclusão crônica, ao uso de dispositivos de aterectomia rotacional e à utilização de fios-guia hidrofílicos.

Em 1994, Ellis et al. ${ }^{1}$ classificaram as perfurações coronárias em uma escala de 1 a 4 , com base em critérios angiográficos (Tabela 1). Estudo subsequente avaliou o desfecho intra-hospitalar de pacientes com

\section{ABSTRACT}

Treatment with the Double Guiding Catheter Technique for Type III Coronary Perforation

Coronary perforation is a rare complication of percutaneous coronary intervention and is potentially catastrophic. Coronary perforation may lead to pericardial effusion with or without cardiac tamponade and if not diagnosed early on and treated properly it is life-threatening. We present a case of percutaneous coronary intervention complicated by coronary perforation, which was quickly treated by the reversal of anticoagulation, prolonged balloon inflation and a covered-stent, using the double guiding catheter technique.

DESCRIPTORS: Percutaneous coronary intervention. Stents. Cardiac catheters.

PC e concluiu que aqueles com grandes perfurações (tipo III) tiveram uma mortalidade de $21,4 \%$ vs. $\leq 1 \%$ comparada às perfurações tipo I ou II. ${ }^{2}$

O objetivo do relato foi descrever o manejo particular de um caso de PC que ocorreu em paciente submetido à ICP eletiva em serviço de cardiologia intervencionista com grande volume de procedimentos, de forma inesperada, após pré-dilatação com balão (relação balão:artéria $<1$ ).

\section{RELATO DO CASO}

Paciente do sexo masculino, 66 anos, com antecedente de hipertensão arterial sistêmica e dislipidemia,

\footnotetext{
Residente do Serviço de Cardiologia Invasiva do Instituto Dante Pazzanese de Cardiologia. São Paulo, SP, Brasil.

2 Residente do Serviço de Cardiologia Invasiva do Instituto Dante Pazzanese de Cardiologia. São Paulo, SP, Brasil.

3 Residente do Serviço de Cardiologia Invasiva do Instituto Dante Pazzanese de Cardiologia. São Paulo, SP, Brasil.

${ }^{4}$ Doutor. Cardiologista intervencionista do Serviço de Cardiologia Invasiva do Instituto Dante Pazzanese de Cardiologia. São Paulo, SP, Brasil. ${ }^{5}$ Doutor. Cardiologista intervencionista do Serviço de Cardiologia Invasiva do Instituto Dante Pazzanese de Cardiologia. São Paulo, SP, Brasil.
}

\footnotetext{
${ }^{6}$ Doutor. Cardiologista intervencionista do Serviço de Cardiologia Invasiva do Instituto Dante Pazzanese de Cardiologia. São Paulo, SP, Brasil. ${ }^{7}$ Livre-docente. Diretor da Divisão de Cardiologia Invasiva do Instituto Dante Pazzanese de Cardiologia. São Paulo, SP, Brasil.
}

Correspondência: Evandro Martins Filho. Rua José Antonio Coelho, 300, apto. 96A - Vila Mariana - São Paulo, SP, Brasil - CEP 04011060 E-mail: evandrofilhobr@hotmail.com

Recebido em: 10/9/2013 • Aceito em: 16/11/2013 
apresentava angina estável grau II, tendo sido encaminhado para angioplastia eletiva após cineangiocoronariografia.

A cinecoronariografia mostrou coronária direita e circunflexa sem lesões, descendente anterior (DA) com uma lesão discreta (30\%) proximal e outra lesão segmentar excêntrica de 90\% em seu terço médio (Figura 1A). Realizamos angiografia coronária quantitativa (ACQ) off-line (CAAS II, Pie Medical Imaging, Maastricht, Holanda) que mostrou diâmetro de referência do vaso alvo de 3,06 mm (Figura 1B).

Exames laboratoriais na avaliação clínica pré-ICP estavam dentro dos limites da normalidade. O paciente recebeu clopidogrel 300 mg na véspera e já estava em uso de ácido acetilsalicílico (AAS) 100 mg/dia.

O planejamento da ICP incluiu pré-dilatação, seguida de implante de suporte vascular bioabsorvível (BVS, Abbott Vascular, Temecula, Estados Unidos), conforme protocolo de ensaio clínico específico. O procedimento foi realizado por via femoral direita com introdutor $6 \mathrm{~F}$, sendo utilizados $100 \mathrm{UI} / \mathrm{kg}$ de heparina não fracionada durante o procedimento, objetivando um tempo de coagulação ativada $(\mathrm{TCA})>250$ segundos. Após cate-

\section{TABELA 1 \\ Classificação de Ellis modificada ${ }^{1,3}$ para perfurações coronárias}

\begin{tabular}{cl}
\hline Tipo I & $\begin{array}{l}\text { Cratera extraluminal, sem extravasamento de contraste } \\
\text { linear que sugira dissecção }\end{array}$ \\
Tipo II & $\begin{array}{l}\text { Blush pericárdico ou miocárdico, com orifício de saída } \\
<1 \mathrm{~mm}\end{array}$ \\
Tipo III & $\begin{array}{l}\text { Franco extravasamento de contraste para o pericárdio por } \\
\text { meio de orifício } \geq 1 \mathrm{~mm} \text { de diâmetro }\end{array}$ \\
Tipo IV & $\begin{array}{l}\text { Perfuração com derramamento de contraste diretamente } \\
\text { para o ventrículo esquerdo, para o seio coronário ou para } \\
\text { outra câmara vascular, excluindo o pericárdio }\end{array}$ \\
\hline
\end{tabular}

terização seletiva do óstio da coronária esquerda com cateter-guia JL 3,5 6 F (Figura 2A), a lesão foi cruzada sem dificuldades com corda-guia 0,014 polegadas BMW $^{\circledR}$ (Abbott Vascular, Temecula, Estados Unidos). Em seguida, foi realizada pré-dilatação, usando cateter balão não complacente Trek $^{\circledR} \mathrm{NC}$ (Abbott Vascular, Temecula, Estados Unidos) de 3,25 x 20 mm até 10 atm, visando a uma relação balão:artéria próxima a 1 (relação de 0,97, avaliada pela ACQ off-line), de acordo com recomendação técnica para implante de BVS (Figura 2B).

A angiografia de controle após pré-dilatação mostrou franco extravasamento de contraste para o pericárdio, através de orifício $\geq 1 \mathrm{~mm}$, indicando PC tipo III, associado à oclusão distal do vaso (Figura 2C). Imediatamente, a área da perfuração foi selada por meio de insuflação prolongada, com baixa pressão (4 atm), usando o mesmo cateter balão da pré-dilatação, por cerca de 20 minutos. Simultaneamente, foi realizada a reversão da anticoagulação com 50 mg de protamina endovenosa com objetivo de atingir um TCA $<150$ segundos.

A ecocardiografia evidenciou derrame pericárdico discreto, sem repercussão hemodinâmica. Devido à intercorrência, foi contraindicado o implante do BVS.

Optou-se por empregar a técnica de duplo cateter-guia ${ }^{4-7}$ para implantar stent farmacológico longo cobrindo todo o segmento de lesão, seguido de implante de stent recoberto com politetrafluoretileno (PTFE) curto intra-stent, apenas no sítio da PC. Para isso, foi obtido um segundo acesso arterial com punção em artéria femoral esquerda e colocação de outro introdutor $6 \mathrm{~F}$. Em seguida, foi utilizado cateter-guia JL 3,5 $6 \mathrm{~F}$ posicionado paralelamente ao primeiro, no óstio da coronária esquerda. Uma corda-guia 0,014" (CholCE ${ }^{\circledR}$ Floppy, Boston Scientific, Natick, Estados Unidos) foi avançada até a borda proximal do cateter balão, que encontrava-se insuflado. De forma rápida e sincroni-
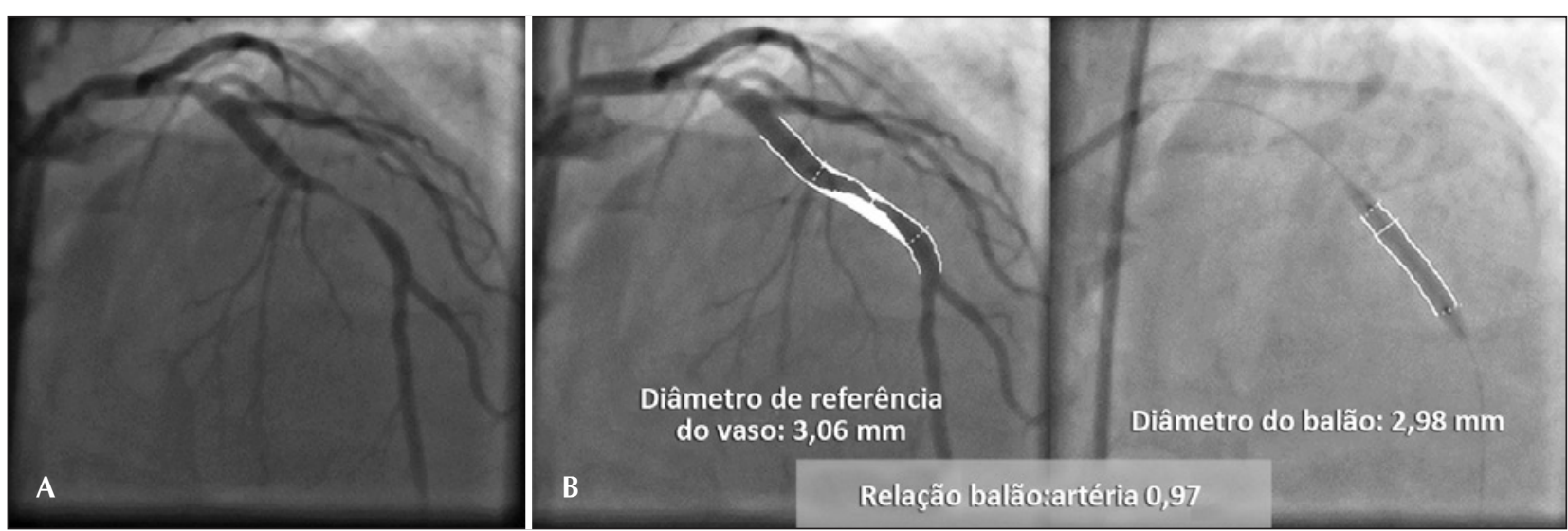

Figura 1. (A) Angiografia de referência pré-procedimento. (B) Angiografia coronária quantitativa antes e durante pré-dilatação mostrando a relação balão:artéria de 0,97 . 
zada, realizou-se a desinsuflação do balão; a segunda corda-guia foi introduzida rapidamente até um ponto distal na DA, e o cateter balão foi insuflado novamente (Figura 2D). Um stent farmacológico Endeavor ${ }^{\circledR} 3 \mathrm{x}$ $24 \mathrm{~mm}$ (Medtronic Inc., Minneapolis, Estados Unidos) foi avançado pela segunda corda-guia, até a borda proximal do cateter balão insuflado e até 10 atm na área da perfuração. Numa manobra rápida, o balão foi desinsuflado e tracionado, e o stent foi avançado e implantado no sítio da perfuração, abrangendo todo o segmento de lesão no terço médio da DA (Figuras $2 \mathrm{E}$ e $2 \mathrm{~F}$ ). Em seguida, um stent revestido Jostent GraftMaster $^{\circledR}$ 3,5 x 12 mm (Abbott Vascular, Temecula, Estados Unidos) foi implantado (liberado com 14 atm) intra-stent no sítio da perfuração, usando a mesma técnica de rápida desinsuflação do balão e implante do stent (Figura 2G e 2G1).

A angiografia de controle evidenciou selamento completo da PC e ausência de lesão residual (Figura $2 \mathrm{H})$.

Ecocardiogramas durante o procedimento e de 3 horas após mostraram derrame pericárdico mínimo; não houve aumento significativo da troponina. O ecocardiograma foi repetido no dia seguinte e não mostrou alterações adicionais. O paciente foi encaminhado para a Unidade Coronária e recebeu alta no segundo dia após o procedimento, com prescrição de terapia antiplaquetária dupla durante 12 meses.

\section{DISCUSSÃO}

Durante a ICP, a PC pode ocorrer como consequência do avanço do fio-guia, balão ou stent, liberação do stent, hiperdimensionamento do stent/balão, fratura do stent ou passagem subintimal do balão/stent, levando a dissecções graves e com perfuração. É relatado que a perfuração após implante de stent é causada principalmente por dilatação excessiva ou implante de stent hiperdimensionado para o vaso tratado.

A principal causa de PC é o manuseio inadequado do fio-guia, quer seja ao cruzar a lesão, posicionando-o inadvertidamente fora do leito arterial, quer seja ao posicioná-lo no leito arterial distal, além do ponto ideal, forçando e perfurando. Fios-guia com revestimento hidrofílico, principalmente aqueles com ponta revestida com polímero, indicados em lesões coronárias muito graves, oclusões e tortuosidades acentuadas, conferem aumento do risco de perfuração devido ao seu baixo coeficiente de atrito e facilidade de migração distal. ${ }^{8-13}$ Apesar de mais frequentes, as PC relacionadas ao trauma pela corda-guia geralmente são facilmente bem controladas, e a ocorrência de derrame pericárdico importante com tamponamento cardíaco é pouco frequente. ${ }^{14}$

O hiperdimensionamento do stent/balão confere risco aumentado para PC durante ICP. No relato de Ajluni et al. ${ }^{15}$, as perfurações foram mais frequentes quando a relação balão:artéria foi de 1,3 \pm 0,3,
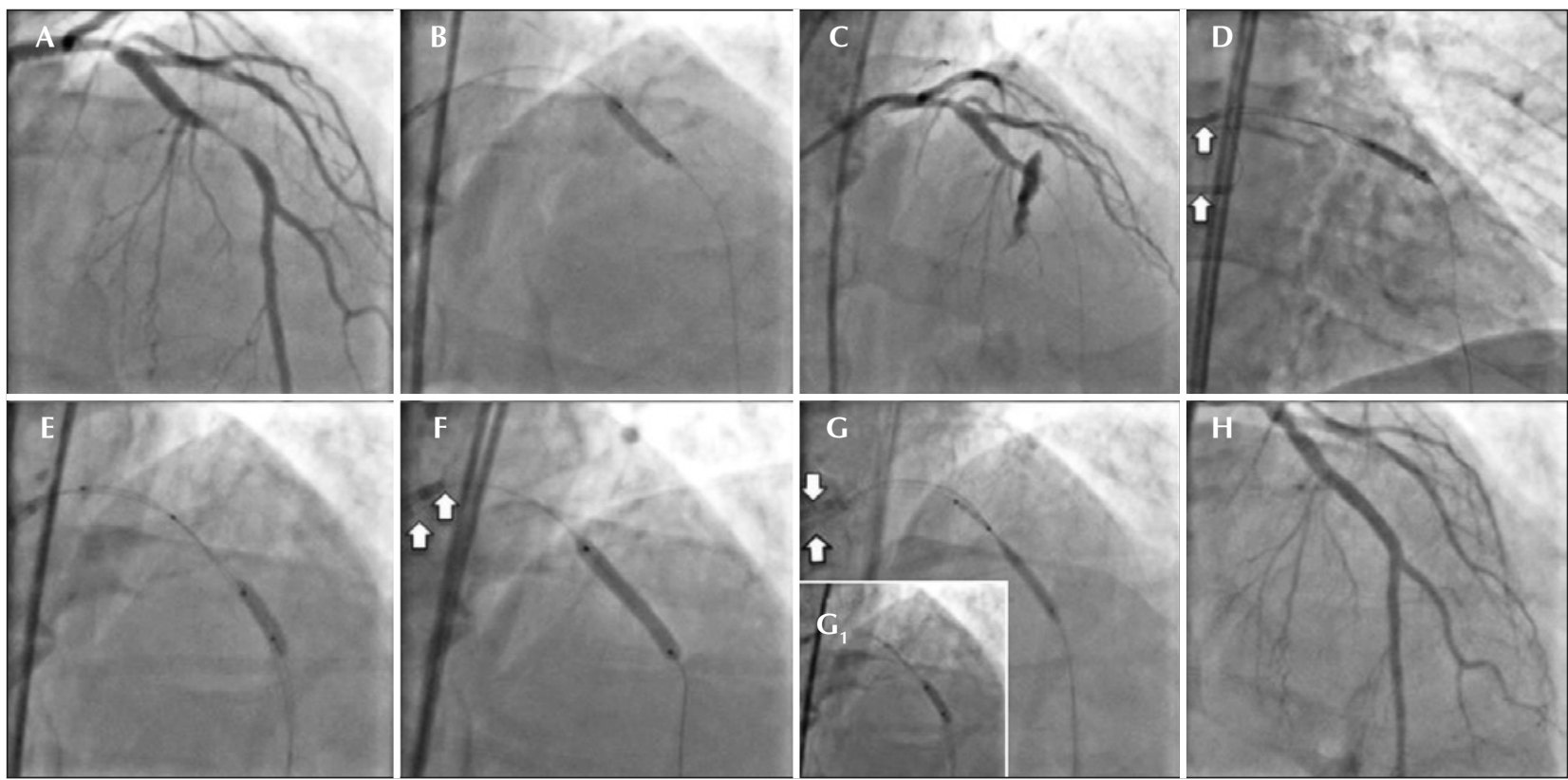

Figura 2. (A) Angiografia coronária em oblíqua anterior esquerda mostrando lesão suboclusiva no terço médio da artéria descendente anterior. (B) Pré-dilatação com cateter balão não complacente Trek NC (Abbott Vascular Inc.). (C) Injeção de controle após pré-dilatação mostrando perfuração coronária do tipo III com extravasamento de contraste e fluxo distal TIMI 0. (D) Insuflação prolongada (20 minutos) do cateter balão, no local da perfuração. (E) Segundo cateter-guia por meio do qual os stents farmacológico e o revestido serão avançados. (F) Liberação de stent farmacológico abrangendo todo o segmento de lesão no terço médio da descendente anterior. (G e G1) Posicionamento e liberação do stent revestido no local da perfuração com rápida manobra de desinsuflação do cateter balão, que permanecia insuflado. (H) Injeção de controle final (setas brancas indicam os dois cateteres-guia) 
comparada às ICP, com relação balão:artéria de 1,0 $\pm 0,3(P<0,001)$. De forma semelhante, Ellis et al. ${ }^{1}$ publicaram um registro de pacientes submetidos à ICP no qual aqueles que sofreram $\mathrm{PC}$ tiveram uma relação balão:artéria de 1,19 $\pm 0,17$ vs. 0,92 $\pm 0,16$ daqueles sem essa complicação $(P=0,03)$. Essa observação foi confirmada em outro grande estudo randomizado, no qual a relação balão:artéria $>$ 1,1 aumentou de duas a três vezes o risco de dissecção grave e perfuração, quando comparada à relação balão:artéria $<1,1 .{ }^{3}$ No caso descrito, a relação balão:artéria foi de 0,97 e, portanto, não justifica a complicação ocorrida (Figura 1).

Silva et al. ${ }^{16}$ relataram a experiência em ocorrência de perfurações coronárias no Instituto Dante Pazzanese de Cardiologia, em São Paulo (SP) entre dezembro de 2007 a janeiro de 2012, na qual 5.585 pacientes foram submetidos à ICP e 18 sofreram PC (0,32\%). Nesse grupo, a artéria DA foi o vaso mais frequentemente tratado $(61,1 \%)$, bem como lesões tipo C $(61,1 \%)$. As oclusões crônicas foram abordadas em 27,8\% dos casos. A maioria das perfurações coronárias tinha uma complexidade inferior, de acordo com a classificação de Ellis modificada. O dispositivo com o cateter balão foi responsável pela perfuração em $61,1 \%$ dos casos. Insuflação prolongada, com um cateter balão e reversão de heparina com protamina, foi realizada em $72,2 \%$ e $88,9 \%$ dos casos, respectivamente. Não houve mortes associadas à PC. Sexo feminino e oclusões crônicas foram preditores de PC, de acordo com a análise multivariada.
No presente caso, a fim de minimizar o tempo entre a retirada do balão insuflado no sítio da PC e a introdução e o implante do stent recoberto, decidiu-se utilizar a técnica de duplo cateter-guia (Figura 3). Essa técnica foi descrita pela primeira vez por Silver et al. ${ }^{6}$ para o tratamento de PC tipo III, selada com stent recoberto com PTFE, que foi liberado por meio de um segundo cateter-guia inserido na artéria femoral contralateral, enquanto o local da perfuração era controlado e selado com o balão de angioplastia. Ben-Gal et al. ${ }^{5}$ descreveram recentemente a primeira série de doentes tratados pela técnica de duplo cateter. O número de pacientes incluídos nesse estudo não foi suficientemente adequado para gerar conclusões definitivas, mas a observação de taxa relativamente mais baixa de eventos adversos utilizando a técnica de duplo cateter-guia parece favorecer essa abordagem.

A técnica permite a preparação e a inserção simultânea e paralela de outro sistema, incluindo cateter-guia, fio-guia e stent recoberto, enquanto a vedação temporária da perfuração é feita por meio do sistema utilizado para a ICP, que resultou na PC. Devido ao perfil elevado, existem limitações na navegabilidade dos stents recobertos com PTFE e, às vezes, dificuldade para chegar até o local da perfuração. Além disso, a luz interna dos cateteres-guia usados habitualmente nas ICP (6 e 7 F) não é suficiente para a passagem de um balão e de um stent recoberto com PTFE paralelamente.

Essa técnica de duplo cateter-guia exige algumas considerações. Primeiramente, em situações de tampo-

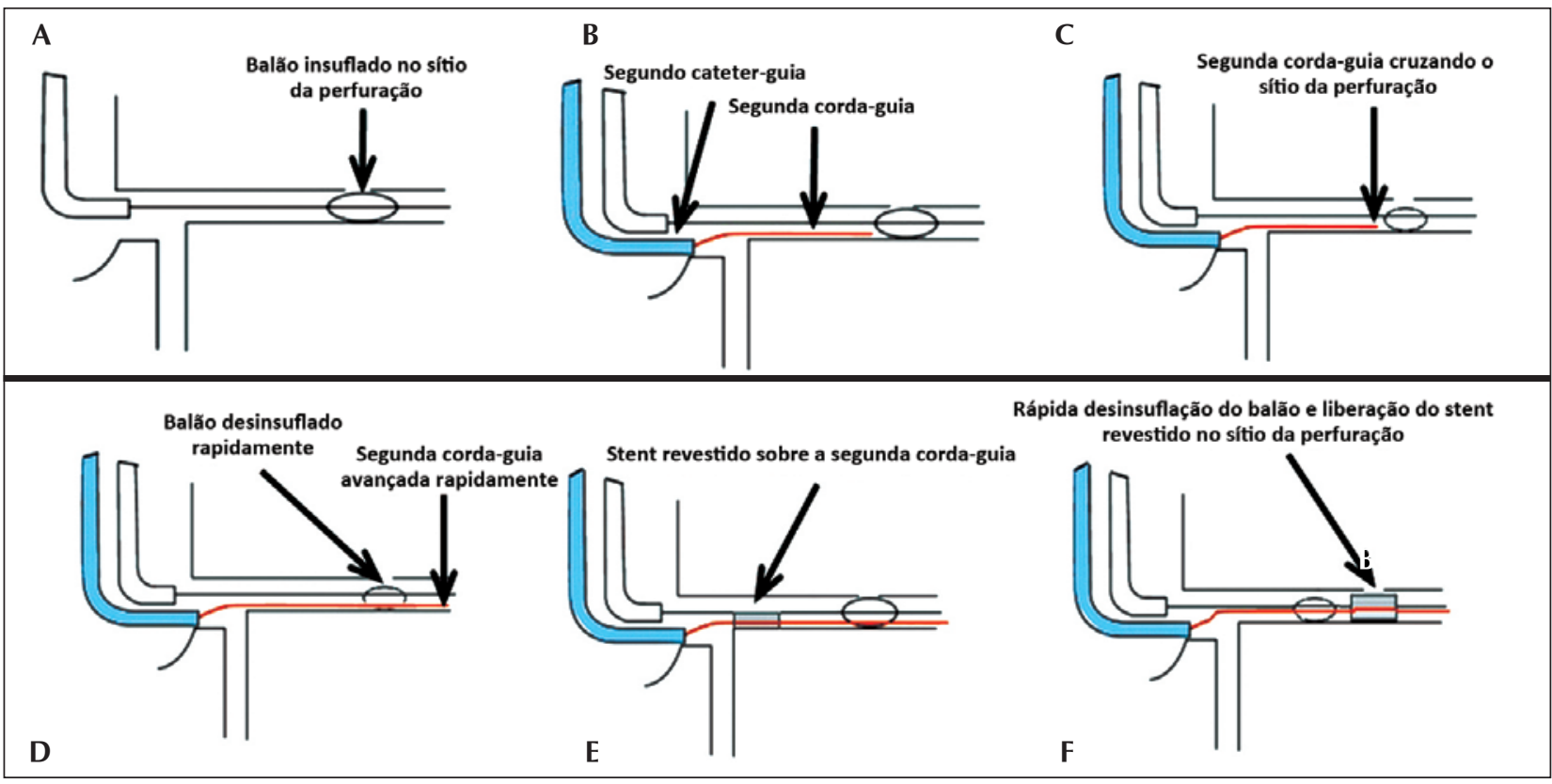

Figura 3. Diagrama esquemático mostrando a técnica de duplo cateter-guia. (A) Balão usado na angioplastia insuflado com baixa pressão (4 atm) no sítio de perfuração. (B) Recuo cuidadoso do primeiro cateter-guia e cateterização do óstio da coronária com o segundo cateter-guia. (C e D) Avanço da segunda corda-guia, cruzando o sítio da perfuração com desinsuflação rápida do balão, permitindo sua passagem. (E) Stent revestido avançando sobre a segunda corda-guia. (F). Rápida manobra de desinsuflação, recuo do balão e liberação do stent revestido no sítio da perfuração. 
namento cardíaco iminente, nas quais um stent revestido pode ser fácil e rapidamente implantado no local da perfuração, o operador pode considerar pericardiocentese e, em seguida, retirar o balão e implantar o stent revestido, mantendo a drenagem contínua do pericárdio. Para tais pacientes, a técnica de duplo cateter-guia pode não ser necessária. Em segundo lugar, o operador deve ter ciência de quando interromper o procedimento e indicar intervenção cirúrgica de emergência. A estabilidade hemodinâmica dificilmente pode ser mantida em doentes com grandes perfurações que envolvam extensas áreas de miocárdio viável. Nesses casos, se um stent recoberto com PTFE não puder ser facilmente implantado, suporte circulatório e intervenção cirúrgica devem ser considerados imediatamente.

O sucesso terapêutico dessa grave complicação está associado à rapidez no controle do derrame pericárdico, uma vez que a mortalidade está intimamente ligada ao desenvolvimento de tamponamento cardíaco. O tratamento requer a detecção precoce e classificação angiográfica da perfuração, a imediata insuflação do balão no ponto da perfuração, a neutralização da heparina, a transfusão de plaquetas em casos associados à utilização de inibidores da glicoproteína Ilb/Illa e o implante de stents recobertos, quando necessário. Para isso, na suspeita de PC durante insuflação de balão/stent com relação balão:artéria $>1,3$, deve-se desinsuflar o balão e rapidamente, sem retirá-lo do local, deve-se fazer angiografia para verificação; se confirmada PC, reinsuflar imediatamente o balão com pressão baixa, checando a oclusão do vaso/PC, com pequena injeção de contraste.

A técnica de duplo cateter-guia pode ser considerada em algumas situações, em especial quando o primeiro cateter-guia está sendo usado para a insuflação persistente do balão, a fim de selar temporariamente a perfuração. Grande dificuldade é prevista para implantar o stent recoberto, devido à existência de tortuosidade, calcificação acentuada ou stent prévio no segmento proximal. Assim, os operadores ganham tempo e facilidade para implantar o stent recoberto com PTFE, proporcionando mais segurança ao procedimento, evitando intervenção cirúrgica e diminuindo mortalidade associada a essa complicação de ICP.

\section{CONFLITO DE INTERESSES}

Os autores declaram não haver conflito de interesses relacionado a este manuscrito.

\section{REFERÊNCIAS}

1. Ellis SG, Ajluni S, Arnold AZ, Popma JJ, Bittl JA, Eigler NL, et al. Increased coronary perforation in the new device era.
Incidence, classification, management, and outcome. Circulation. 1994;90(6):2725-30.

2. Witzke CF, Martin-Herrero F, Clarke SC, Pomerantzev E, Palacios IF. The changing pattern of coronary perforation during percutaneous coronary intervention in the new device era. J Invasive Cardiol. 2004;16(6):257-301.

3. Ellis SG, Vandormael MG, Cowley MJ, DiSciascio G, Deligonul U, Topol EJ, et al. Coronary morphologic and clinical determinants of procedural outcome with angioplasty for multivessel coronary disease: implications for patient selection. Multivessel Angioplasty Prognosis Study Group. Circulation. 1990;82(4):1193-202.

4. Barbosa RR, Costa R, Slhessarenko JR, Coelho FM, Feres F. Tratamento de perfuração coronária tipo IV durante intervenção coronária percutânea. Rev Bras Cardiol Invasiva. 2013;21(1):73-7.

5. Ben-Gal Y, Weisz G, Collins MB, Genereux P, Dangas GD, Teirstein PS, et al. Dual catheter technique for the treatment of severe coronary artery perforations. Catheter Cardiovasc Interv. 2010;75(5):708-12.

6. Silver KH, Bauman WB, Berkovitz KE. Dual-catheter covered stenting: a novel approach to the treatment of large coronary artery perforations. J Invasive Cardiol. 2003;15(6):348-50.

7. Ziakas A, Economou F, Feloukidis C, Kiratlidis K, Stiliadis I. Left anterior descending artery perforation treated with graft stenting combining dual catheter and side branch graft stenting techniques. Herz. 2012;37(8):913-6.

8. Dixon SR, Webster MW, Ormiston JA, Wattie WJ, Hammett CJ. Gelfoam embolization of a distal coronary artery guidewire perforation. Catheter Cardiovasc Interv. 2000;49(2):214-7.

9. Gruberg L, Pinnow E, Flood R, Bonnet Y, Tebeica M, Waksman $\mathrm{R}$, et al. Incidence, management, and outcome of coronary artery perforation during percutaneous coronary intervention. Am J Cardiol. 2000;86(6):680-2

10. Dippel EJ, Kereiakes DJ, Tramuta DA, Broderick TM, Shimshak TM, Roth EM, et al. Coronary perforation during percutaneous coronary intervention in the era of abciximab platelet glycoprotein Ilb/IIla blockade: an algorithm for percutaneous management. Catheter Cardiovasc Interv. 2001;52(3):279-86.

11. Stankovic G, Orlic D, Corvaja N, Airoldi F, Chieffo A, Spanos $\mathrm{V}$, et al. Incidence, predictors, in-hospital, and late outcomes of coronary artery perforations. Am J Cardiol. 2004;93(2):213-6.

12. Shimony A, Zahger D, Van Straten M, Shalev A, Gilutz H, Ilia $\mathrm{R}$, et al. Incidence, risk factors, management and outcomes of coronary artery perforation during percutaneous coronary intervention. Am J Cardiol. 2009;104(12):1674-7

13. Shimony A, Joseph L, Mottillo S, Eisenberg MJ. Coronary artery perforation during percutaneous coronary intervention: a systematic review and meta-analysis. Can J Cardiol. 2011; 27(6):843-50.

14. Teis A, Fernandez-Nofrerias E, Rodriguez-Leor O, Tizon $\mathrm{H}$, Salvatella $N$, Valle $V$, et al. Coronary artery perforation by intracoronary guide wires: risk factors and clinical outcomes. Rev Esp Cardiol. 2010;63(6):730-4.

15. Ajluni SC, Glazier S, Blankenship L, ONeill WW, Safian RD. Perforations after percutaneous coronary interventions: clinical, angiographic, and therapeutic observations. Cathet Cardiovasc Diagn. 1994;32(3):206-12.

16. Silva WA, Costa RA, Campostrini T, Costa Jr. JR, Siqueira DA, Staico R, et al. Incidência, manejo e prognóstico de perfurações coronárias. Rev Bras Cardiol Invasiva. 2012;20(3):295-302. 